\title{
The Role of the Microbiome in Age-Related Macular Degeneration: A Review of the Literature
}

\author{
Athanasios Zisimopoulos $^{\mathrm{a}} \quad$ Olga Klavdianou $^{\mathrm{a}}$ Panagiotis Theodossiadis $^{\mathrm{b}}$ \\ Irini Chatziralli ${ }^{b}$ \\ ${ }^{a}$ Medical School, National and Kapodistrian University of Athens, Athens, Greece; ${ }^{\text {b }}$ 2nd Department of \\ Ophthalmology, Attikon University Hospital, National and Kapodistrian University of Athens, Athens, Greece
}

\section{Keywords}

Age-related macular degeneration · Diagnosis .

Microbiome · Treatment

\section{Abstract}

Background: Age-related macular degeneration (AMD) is a progressive, multifactorial, degenerative disease and the leading cause of severe visual loss in the elderly population. The exact pathogenesis of AMD remains elusive, being the combination of genetic, environmental, metabolic, and functional processes. A better understanding of the disease's pathophysiology can lead to new treatment targets. The human microbiome seems to be a potential therapeutic pathway for AMD, as it has been recently proven to play a role in its pathogenesis. Summary: This review sheds light on the association between the microbiome and AMD. Key Messages: The current evidence based on the existing literature shows that there are differences in taxonomical and functional profiles in the human microbiome between patients with AMD and controls, suggesting that the microbiome is implicated in AMD onset and progression, being a link between AMD and nutrition/diet. Additionally, specific bac-

karger@karger.com

(c) 2021 S. Karger AG, Basel

www.karger.com/oph

Karger $\stackrel{2}{=}$ terial classes have been proposed as potential biomarkers for AMD diagnosis. Further randomized clinical studies with a large sample are needed to elucidate the role of the microbiome in AMD and to draw more solid conclusions.

(c) 2021 S. Karger AG, Basel

\section{Introduction}

Age-related macular degeneration (AMD) is a complex, multifactorial, progressive degenerative disease and is the leading cause of severe visual loss in the elderly population in the industrialized world $[1,2]$. Despite the investigation of new pathophysiological pathways and new treatment modalities, AMD affects about 30-50 million people globally and it is expected to increase 10 -fold by 2040 [2]. The prevalence of AMD increases with age, ranging from $0.2 \%$ (for people aged $55-64$ years) to $13 \%$ (for those older than 85 years) [1]. AMD, in the early stages, is characterized by drusen formation and pigmentary

\section{A.Z. and O.K. contributed equally to this work.}

Irini Chatziralli

Department of Ophthalmology

Attikon University Hospital, 1 Rimini Street

GR-12462 Haidari (Greece)

eirchat@yahoo.gr 
changes in the retinal macular area without visual impairment. However, it may progress to the advanced dry form with significant loss of retinal pigment epithelium (RPE), known as "geography atrophy," or to choroidal neovascularization development (neovascular AMD), both resulting in moderate to severe visual loss [3]. Interestingly, it is unclear whether these forms are distinct or if they are different features of the same spectrum [4].

The exact pathogenetic mechanism of AMD remains elusive. It is thought to be the result of the combination of genetic, environmental, metabolic, and functional processes [5]. Residual bodies produced by RPE cells, which are typically cleared by the choriocapillaris, accumulate with aging, due to the inability of the RPE to operate normally, while changes to the functionality of Bruch's membrane are also involved. Thus, the material pools between the 2 layers, producing the phenotype of drusen deposits [6]. The latter potentiate RPE and Bruch's membrane dysfunction and induce photoreceptor loss and the stimulation of angiogenic factors, eventually leading to endstage AMD [7].

Risk factors known for AMD development consist of demographic characteristics, environmental and nutritional factors, as well as genetics [8-10]. Age is considered the most important demographic risk factor, while smoking and sunlight exposure are also known to be aggravating environmental factors. Moreover, body weight is associated with the progression of AMD, which has been studied and confirmed via measurements of body weight, such as body mass index, waist-to-hip ratio, and waist circumference. It is worth noting that dietary habits are thought to either prevent or accelerate the disease progression. Diets consisting mainly of fruits, vegetables, legumes, grains, and nuts, have been found to have a beneficial effect, while consumption of a high-glycemic index diet is considered a risk factor for disease development and progression [8]. In addition, genetic loci which can contribute to the initiation and progression of AMD have been established. Nevertheless, merely their presence is not sufficient for the development of AMD, suggesting the interference of environmental factors as a key element in its pathogenesis [11]. The most commonly reported genes whose variants are associated with AMD are the complement factor $\mathrm{H}(\mathrm{CFH})$ gene and the ARMS2/ HTRA1 gene [12-14]. Other risk factors include oxidative stress and various comorbidities, like hypertension, chronic kidney disease, hyperthyroidism, and diabetes mellitus [8].

The need for a better understanding of the pathophysiology to establish new targets for treatment has be- come apparent. The human microbiome poses an enticing prospect as a potential therapeutic pathway for AMD, as it can have a pivotal role in its pathogenesis. The microbiome refers to a community of microorganisms and all of its genetic material. Of note, the human microbiota consists of the collection of microorganisms, which includes bacteria, viruses, archaea, and Eukarya that colonize the human body, most noteworthy being the gut microbiota. The microbiome plays a major role in food digestion and affects the metabolism of their host. Additionally, its composition may modulate the immune response and take part in the host's immune system by posing as a competitor to opportunistic pathogens. However, an imbalance in the microorganisms' load, triggered by smoking, diet, or other environmental factors, can lead to situations associated with eye pathology [15-17]. The purpose of this review is to scrutinize the current literature and summarize evidence regarding the implication of the human microbiome in AMD.

\section{The Potential Association between Microbiota and AMD}

It is widely known that inflammation is involved in the development of AMD. Of note, inflammatory molecules, prothrombin, HLA-DR, and complement proteins have been detected in drusen in early AMD, while macrophages and lymphocytes have also been found in AMD lesions [8]. Gut microbiota has been supported to enhance the inflammation process. To understand the proposed mechanism by which gut microbiota may contribute to ocular para-inflammation, one should consider the normal gut function. The intestinal mucosal system is equipped with toll-like receptors (TLR) and nod-like receptors (NLR), which can recognize bacteria and their products, inducing host defense gene expression to protect against potential pathogen microorganisms. This is mainly achieved through the activation of the NF- $\kappa B$ pathway, a proinflammatory and immune cell recruitment signal. A healthy gut microbiota is essential in maintaining the human body in a homeostatic state. When the microbiota homeostasis has failed to be maintained, under state of aberrant microbial composition or function (dysbiosis), the ratio of Firmicutes/Bacteroidetes increases, causing thinning of the intestinal mucus layer, local tissue damage, and disruption of the gut-vascular barrier with consequent intestinal permeability. In this way, commensal bacteria and byproducts, including 
lipopolysaccharides among other pathogen-associated pattern molecules (PAMPs), enter into the blood circulation or lymphatics, where they travel to tissues, resulting in dysregulation of the extraintestinal adaptive immune response [18-22]. This state of systemic low-grade inflammation and metabolic endotoxemia activates the proinflammatory signaling by the pattern recognition receptors of the TLR of microglia and cells of the RPE, also inducing ocular inflammation [23, 24]. Another possible mechanism is the molecular mimicry model, which includes the cross-reactivity of self-antigens with intestinal commensal peptides, leading to tissue-reactive T-cell activation, which promotes disease [22]. Finally, a T-cell threshold model, describing a situation in which a relative dysbiosis of the intestinal microbiome could result in maladaptive immuno-differentiation, has been proposed [22].

Furthermore, interdependency of the functional or taxonomic profile of the commensal microbiota and the expression of the complement factor $\mathrm{CFH}$ gene or the deregulation of the host complement system may be a potential mechanism that could be linked with AMD $[25,26]$. Purine signaling pathway genes have been found to be correlated with an unstable immune system response, as seen in autoimmune diseases [27]. The genes facilitating the aforementioned pathway were found to have augmented expression in patients suffering from AMD compared to controls. Moreover, ZyssetBurri et al. [26] examined the association between the intestinal microbiome and the complement system in neovascular AMD and found that single nucleotide polymorphisms in the $\mathrm{CFH}$ gene associated with $\mathrm{AMD}$ were positively correlated with Negativicutes, a proposed biomarker for AMD. A cause-and-effect relationship remains unclear; however, the intestinal microbiome may potentiate an aberrant complement activation via purine signaling pathways that aid to the development of AMD. Specifically, the intestinal microbiome of C3-deficient mice was reported to be enriched in genes of the 5-aminoimidazole ribonucleotide biosynthesis pathways, which may also be involved in the pathogenesis of AMD, pointing out that this pathway may be a key point in the interconnection between the intestinal microbiome, the complement system, and AMD [26]. In addition, the complement system is a key part of the innate system, operating through TLR signaling. Therefore, when overactivated or deregulated, the complement can be a major link between infection and inflammation, as in the case of AMD [23, 26].

\section{Results of Preclinical and Clinical Studies regarding Microbiota and AMD}

\section{The Role of Diet in Gut Microbiota Modulation and} AMD Development

Three preclinical studies have investigated the effects of a high-fat or high-glycemic diet on the composition of gut microbiota and on disease development and progression $[11,28,29]$. In all studies both diets worked as aggravating factors for disease's characteristics. Noticeably, Andriessen et al. [11] reported an exacerbation of choroidal neovascularization in high-fat diet-fed mice compared to regular diet-fed mice. Moreover, Rowan and Taylor [28] found that several advanced AMD characteristics, such as outer nuclear layer thinning, inner retinal layer thinning, photoreceptor disorganization and degeneration, swelling of inner segments, loss of synaptic pruning of rod bipolar cell dendrites, subretinal deposits, and RPE cell hypopigmentation, were observed either exclusively or earlier in high-glycemic diet-fed mice than in low-glycemic diet-fed mice. Other factors that are known to connect the gut microbiome and the pathophysiology of AMD were also observed in the high-fat diet-fed mice. In this group, alterations in gut microbiota were accompanied by increased activation of microglia and macrophages during the exudative phase of the disease, while elevated intestinal permeability and increased serum concentrations of inflammatory cytokines were also reported [11]. The gut microbiota composition was also found to differ in mice with high-fat diet-induced AMD compared to healthy controls, as there was a shift of the ratio of Bacteroidetes/Firmicutes, with an increase of the Firmicutes population [11, 28]. Rowan et al. [29] further showed that there was an association of high-glycemic diet-induced AMD with individual taxonomic units and specifically with 2 families of the Clostridiales order, Ruminococcaceae and Lachnospiraceae. Classes, such as Bacteroidales and Erysipelotrichia, which are considered to be protective against AMD were found to be enriched in low-glycemic diet-fed mice [28]. When the gut microbiota was restored in all studies, either with antibiotic treatment (neomycin) and microbiota transplantation [11] or with a switch to a low-glycemic diet [28], the aforementioned disease characteristics and pathogenic factors were also arrested or reversed.

\section{Nasal, Oral, Pharyngeal, and Intestinal Microbiota in} AMD Patients

When comparing the microbiome of AMD patients to that of healthy controls, several studies reported notable differences [26, 30-32]. As far as the nasal and oral mi- 
crobial communities are concerned, Rullo et al. [30] found statistically significant differences between cases of neovascular AMD and healthy controls. Specifically, in the nasal microbial communities, Streptococcus, Burkholderiales, Actinomycetaceae, Gemella, Proteobacteria, Actinomyces, and Veillonella were all found to be significantly increased in AMD patients over controls, while members of the Clostridia class were enriched in controls [30].

In the oral microbiota, communities of Propionibacteriales, Rothia, Staphylococcus, and Cornyebacteriaceae were higher in patients with AMD over controls, while Fusobacterium and Bacilli were higher in controls over cases [30]. Gemella and Streptococcus were also found to be more abundant in the pharyngeal microbiome of AMD patients compared to healthy controls in a recent study by Ho et al. [31], while Prevotella and Leptotrichia were reported to have an opposite trend, being higher in controls. In the same study, when observing the pharyngeal microbiota of early versus advanced cases of AMD, no statistically significant differences were found [31].

Regarding the intestinal microbiome, Lin [32] mentioned that in patients with advanced AMD, Prevotella was in higher abundance, while Ruminococcaceae and Rikenellaceae bacteria were lower compared to controls. However, Zinkernagel et al. [33] found that in neovascular AMD cases there was a higher abundance of the genera Anaerotruncus and Oscillibacter, as well as Ruminococcus torques and Eubacterium ventriosum. Zysset-Burri et al. [26] also reported that the class Negativicutes (Firmicutes species) was enriched in patients with neovascular AMD and can serve as a potential biomarker for AMD, but the genus Oscillibacter was more prominent in controls. Both studies noted that Bacteroides species were higher in controls, suggesting that they may be protective for neovascular AMD [26, 33]. As far as the functional characteristics of AMD patients' intestinal microbiota are concerned, their microbiota was enriched in genes of the purine ribonucleosides degradation pathway and in genes of the L-alanine fermentation, glutamate degradation, and arginine biosynthesis pathways, while they were decreased in genes of the fatty acid elongation pathway [26, 33].

In an effort to determine whether the trends in microbial abundances were correlated with risk factors for AMD, Zysset-Burri et al. [26] reported that the order Clostridiales and the class Negativicutes positively correlated with $\mathrm{CFH} 3$, while Clostridiales negatively correlated with $\mathrm{CFH} 1$ and $\mathrm{CFH} 2$ variants. The same group examined whether AMD patients could be identified based on their gut microbiotas' characteristics and concluded that 7 features, including the class Negativicutes, the order Selenomonadales, and the species Phascolarctobacterium, Bacteroides cellulosilyticus, Sutterella wadsworthensis, Bifidobacterium longum, and Bacteroides caccae, could be used in the future as potential biomarkers [26].

\section{Therapeutic and Future Implications}

Intestinal microbiota could be a target of treatment strategies in ocular diseases having an inflammatory component, such as AMD. First of all, long-term dietary modifications or administration of oral probiotics, which could induce beneficial bacteria to produce metabolites, promoting immune homeostasis and reduce complement activation, could be recommended. Additionally, causative bacteria could be eliminated using antibiotics. A final approach might be to supplant an entire community of intestinal bacteria with a normal community, using fecal microbial transplant, although it is challenging to determine the appropriate donor to avoid transplant complications $[11,22]$. Further investigation could aim at modifying the intestinal microbiome in neovascular AMD patients, targeting the decrease of the Firmicutes/ Bacteroides ratio, trying to potentially regulate the complement pathway as well.

\section{Discussion}

Understanding the pathophysiology of AMD is a significant step towards establishing a better treatment regimen and improved outcomes. The role of the microbiome in AMD emerges as a potential diagnostic and therapeutic target. As multiple studies reported, differences in taxonomic and functional profiles are present when comparing healthy controls to patients with AMD, suggesting a possible role of certain microbial communities in the disease onset and progression [31]. These findings may indicate a potential prophylactic role of specific microbial communities in AMD development and progression by modulating the host's immune response to pathogens [33]. Of great interest is the fact that alterations in microbiota due to antibiotics or probiotics administration without diet alteration may affect the deleterious effects in AMD progression, exhibiting how the microbiota can influence ocular pathology in a diet-independent manner [11]. Based on the latter, it may be suggested that long-term dietary intervention may allow modulation of 
an individual's microbiome to avoid disease onset and/or progression, pointing out the direction of individualized treatment in the future.

An interesting point that should be mentioned is the use of certain taxonomical and functional classes, that is Negativicutes, as a biomarker for AMD diagnosis, being a cornerstone for potential new diagnostic tools for AMD [26]. Since $16 \mathrm{~S} r \mathrm{RNA}$ shotgun sequencing can provide the clinician with an AMD diagnosis, certain bacterial metabolites or byproducts, traceable in routine laboratory work-up, may also lead to such a diagnosis, ideally before AMD is clinically obvious [26]. Promising molecules or substances may be serotonin, certain cytokines, or other PAMPs $[18,19]$.

It is also worthy to note that the correlation of certain commensal microbiota composition and $\mathrm{CFH}$ polymorphisms creates an exciting prospect [26]. However, it has to be determined whether the altered complement pathway is what shapes the composition of the gut flora, or if these bacteria have the ability to cause epigenetic changes or escape complement activation in other ways. Potential limitations of the examined studies are the limited number of participants enrolled, the interaction of confounding factors (obesity, smoking), and the lack of longitudinal data.

\section{Conclusion}

The human microbiome seems to be a potential therapeutic pathway for AMD, since it has been proven to play a role in its pathogenesis. Specifically, differences in taxonomical and functional profiles have been found between patients with AMD and controls, suggesting that the microbiome may be implicated in AMD onset and progres- sion and identifying it as a new treatment target for AMD, being a link between AMD and diet/nutrition. In addition, some authors have indicated the use of certain bacterial classes as biomarkers for AMD diagnosis. A better understanding of the underlying mechanisms affecting the connection between AMD and the microbiome may help clinicians to develop new models of personalized diagnosis and care, based on specific biomarkers and modulation of the human microbiome. Further randomized clinical studies with large samples are needed to elucidate the role of the microbiome in AMD and to draw more solid conclusions.

\section{Statement of Ethics}

This is a review of the literature and Institutional Review Board approval was not needed.

\section{Conflict of Interest Statement}

The authors declare no conflicts of interest.

\section{Funding Sources}

There are no funding sources to declare.

\section{Author Contributions}

O.K. and A.Z. conceived the idea, collected data, analyzed and interpreted data, and drafted the manuscript. P.T. interpreted data and revised the manuscript. I.C., as supervisor, conceived the idea, analyzed and interpreted data, and critically revised the manuscript. All authors have read and approved the final version of the manuscript.

\section{References}

1 Klein R, Peto T, Bird A, Vannewkirk MR. The epidemiology of age-related macular degeneration. Am J Ophthalmol. 2004 Mar;137(3): 486-95.

2 Wong WL, Su X, Li X, Cheung CM, Klein R, Cheng CY, et al. Global prevalence of age-related macular degeneration and disease burden projection for 2020 and 2040: a systematic review and meta-analysis. Lancet Glob Health. 2014 Feb;2(2):e106-16.

3 Ferris FL 3rd, Wilkinson CP, Bird A, Chakravarthy U, Chew E, Csaky K, et al.; Beckman Initiative for Macular Research Classification Committee. Clinical classification of age-related macular degeneration. Ophthalmology. 2013 Apr;120(4):844-51.
4 de Jong PT. Age-related macular degeneration. N Engl J Med. 2006 Oct;355(14):147485.

5 Kijlstra A, Berendschot TT. Age-related macular degeneration: a complementopathy? Ophthalmic Res. 2015;54(2):64-73.

6 Green WR, McDonnell PJ, Yeo JH. Pathologic features of senile macular degeneration. Ophthalmology. 1985 May;92(5):615-27.

7 Biesemeier A, Taubitz T, Julien S, Yoeruek E, Schraermeyer U. Choriocapillaris breakdown precedes retinal degeneration in age-related macular degeneration. Neurobiol Aging. 2014 Nov;35(11):2562-73.
8 Heesterbeek TJ, Lorés-Motta L, Hoyng CB, Lechanteur YT, den Hollander AI. Risk factors for progression of age-related macular degeneration. Ophthalmic Physiol Opt. 2020 Mar;40(2):140-70.

9 Zhang QY, Tie LJ, Wu SS, Lv PL, Huang HW, Wang WQ, et al. Overweight, Obesity, and Risk of Age-Related Macular Degeneration. Invest Ophthalmol Vis Sci. 2016 Mar;57(3): 1276-83.

10 Adams MK, Simpson JA, Aung KZ, Makeyeva GA, Giles GG, English DR, et al. Abdominal obesity and age-related macular degeneration. Am J Epidemiol. 2011 Jun;173(11): 1246-55. 
11 Andriessen EM, Wilson AM, Mawambo G, Dejda A, Miloudi K, Sennlaub F, et al. Gut microbiota influences pathological angiogenesis in obesity-driven choroidal neovascularization. EMBO Mol Med. 2016 Dec;8(12): 1366-79.

12 Boon CJ, Klevering BJ, Hoyng CB, ZonneveldVrieling MN, Nabuurs SB, Blokland E, et al. Basal laminar drusen caused by compound heterozygous variants in the $\mathrm{CFH}$ gene. Am J Hum Genet. 2008 Feb;82(2):516-23.

13 Joachim N, Mitchell P, Rochtchina E, Tan AG, Wang JJ. Incidence and progression of reticular drusen in age-related macular degeneration: findings from an older Australian cohort. Ophthalmology. 2014 Apr;121(4): 917-25.

$14 \mathrm{Wu}$ J, Sun X. Complement system and agerelated macular degeneration: drugs and challenges. Drug Des Devel Ther. 2019 Jul;13: 2413-25.

15 Blum HE. The human microbiome. Adv Med Sci. 2017 Sep;62(2):414-20.

16 Lloyd-Price J, Mahurkar A, Rahnavard G, Crabtree J, Orvis J, Hall AB, et al. Strains, functions and dynamics in the expanded $\mathrm{Hu}$ man Microbiome Project. Nature. 2017 Oct; 550(7674):61-6.

17 Wang B, Yao M, Lv L, Ling Z, Li L. The Human Microbiota in Health and Disease. Engineering. 2017;3(1):71-82.

18 Chassaing B, Gewirtz AT. Gut microbiota, low-grade inflammation, and metabolic syndrome. Toxicol Pathol. 2014 Jan;42(1):49-53.
19 Cani PD, Bibiloni R, Knauf C, Waget A, Neyrinck AM, Delzenne NM, et al. Changes in gut microbiota control metabolic endotoxemiainduced inflammation in high-fat diet-induced obesity and diabetes in mice. Diabetes. 2008 Jun;57(6): 1470-81.

20 Floyd JL, Grant MB. The Gut-Eye Axis: Lessons Learned from Murine Models. Ophthalmol Ther. 2020 Sep;9(3):499-513.

21 Kalyana Chakravarthy S, Jayasudha R, Sai Prashanthi G, Ali MH, Sharma S, Tyagi M, et al. Dysbiosis in the Gut Bacterial Microbiome of Patients with Uveitis, an Inflammatory Disease of the Eye. Indian J Microbiol. 2018 Dec;58(4):457-69.

22 Lin P. Importance of the intestinal microbiota in ocular inflammatory diseases: A review. Clin Exp Ophthalmol. 2019 Apr;47(3):418-22.

23 Chen $\mathrm{M}, \mathrm{Xu} \mathrm{H}$. Parainflammation, chronic inflammation, and age-related macular degeneration. J Leukoc Biol. 2015 Nov;98(5): 713-25.

24 Chaiwiang N, Poyomtip T. Microbial dysbiosis and microbiota-gut-retina axis: the lesson from brain neurodegenerative diseases to primary open-angle glaucoma pathogenesis of autoimmunity. Acta Microbiol Immunol Hung. 2019 Dec;66(4):541-58.

25 Zhang MX, Zhao XF, Ren YC, Geng TT, Yang $\mathrm{H}$, Feng T, et al. Association between a functional genetic polymorphism (rs2230199) and age-related macular degeneration risk: a meta-analysis. Genet Mol Res. 2015 Oct;14(4): 12567-76.
26 Zysset-Burri DC, Keller I, Berger LE, Largiadèr $\mathrm{CR}$, Wittwer $\mathrm{M}$, Wolf $\mathrm{S}$, et al. Associations of the intestinal microbiome with the complement system in neovascular age-related macular degeneration. NPJ Genom Med. 2020 Sep;5(1):34

27 Longhi MS, Moss A, Jiang ZG, Robson SC. Purinergic signaling during intestinal inflammation. J Mol Med. 2017 Sep;95(9):915-25.

28 Rowan S, Taylor A. Gut microbiota modify risk for dietary glycemia-induced age-related macular degeneration. Gut Microbes. 2018; 9(5):452-7.

29 Rowan S, Jiang S, Korem T, Szymanski J, Chang ML, Szelog J, et al. Involvement of a gut-retina axis in protection against dietary glycemia-induced age-related macular degeneration. Proc Natl Acad Sci USA. 2017 May;114(22):E4472-81.

30 Rullo J, Far PM, Quinn M, Sharma N, Bae S, Irrcher I, et al. Local oral and nasal microbiome diversity in age-related macular degeneration. Sci Rep. 2020 Mar;10(1):3862.

31 Ho EX, Cheung CM, Sim S, Chu CW, Wilm $A$, Lin $\mathrm{CB}$, et al. Human pharyngeal microbiota in age-related macular degeneration. PLoS One. 2018 Aug;13(8):e0201768.

32 Lin P. The role of the intestinal microbiome in ocular inflammatory disease. Curr Opin Ophthalmol. 2018 May;29(3):261-6.

33 Zinkernagel MS, Zysset-Burri DC, Keller I, Berger LE, Leichtle $\mathrm{AB}$, Largiadèr $\mathrm{CR}$, et al. Association of the Intestinal Microbiome with the Development of Neovascular AgeRelated Macular Degeneration. Sci Rep. 2017 Jan;7(1):40826. 